\title{
Serotonin 2A receptor gene (HTR2A) polymorphism in alcohol-dependent patients
}

\author{
Małgorzata Wrzosek ${ }^{1}$, Andrzej Jakubczyk ${ }^{2}$, Michał Wrzosek ${ }^{3}$, \\ Halina Matsumoto ${ }^{2}$, Jacek Łukaszkiewicz ${ }^{4}$, Kirk J. Brower ${ }^{5}$, Marcin Wojnar2,5 \\ ${ }^{1}$ Department of Pharmacogenomics, ${ }^{2}$ Department of Psychiatry, ${ }^{3}$ Department of Internal Medicine and \\ Diabetology, ${ }^{4}$ Department of Biochemistry and Clinical Chemistry, Medical University of Warsaw, \\ Żwirki i Wigury 61, PL 02-091 Warszawa, Poland \\ ${ }^{5}$ Department of Psychiatry, University of Michigan, 4250 Plymouth Rd., Ann Arbor, MI 48109, USA
}

Correspondence: Marcin Wojnar, e-mail: marcin.wojnar@wum.edu.pl

\begin{abstract}
:
Background: The serotonergic (5-HT) dysfunction has been frequently described in subjects with alcohol dependence (AD). In the present study, a potential relationship between T102C polymorphism in the 5-HT receptor subtype 2A gene (HTR2A) and alcohol dependence was examined.

Methods: Genotypes were analyzed in 150 AD patients diagnosed with DSM-IV criteria and in 80 healthy controls.

Results: The genetic analysis showed that the frequency of $102 \mathrm{C}$ allele and $\mathrm{C} 102 \mathrm{C}$ genotype in $\mathrm{AD}$ subjects was significantly higher than in controls. Moreover, $\mathrm{AD}$ patients homozygous for $\mathrm{C}$ allele had significantly lower age at onset of alcohol problems than subjects having at least one T allele.

Conclusion: The results suggest a potential role of the T102C HTR $2 A$ polymorphism in development of alcohol dependence.
\end{abstract}

Key words:

alcohol dependence, genetic polymorphism, HTR2A

\section{Introduction}

Alcohol dependence constitutes a serious individual and social problem, which is strongly associated with post-treatment relapse. Although the estimated contribution of genetic factors to alcohol dependence ranges from 40 to $60 \%$, the specific genes and their exact roles have not been identified [4]. In this study selection of a gene that modulates the risk to development of alcohol addiction was based on the premise that serotonergic (5-HT) dysfunction causing depressed mood, anxiety or impulsiveness may predispose to alcohol dependence. Serotonergic neurons of the raphe nuclei affect mood, aggression, sleep, appetite and the development of tolerance to alcohol [3, 8]. There is also evidence that the variations in brain 5-HT synthesis and 5-HT receptor binding are associated with altered neurotransmitter function, plasma cortisol levels, and noradrenergic activity [11, 20]. Furthermore, a number of stress-related psychiatric disorders and alcohol-related disorders are often accompanied by alterations in the serotonergic system $[7,12,13]$. This has been recently confirmed in an animal model by Crawford et al. [3], who found that 5-HT neurons play a unique role in adaptive responses to stress. 
The 5-HT receptor subtype $2 \mathrm{~A}$ gene (HTR2A) is located on chromosome 13 (13q14-q21) and contains the T102C (rs6313) single nucleotide polymorphism. Studies in human brain tissue reported that the expression of the $102 \mathrm{C}$ allele at both mRNA and protein levels in the temporal cortex is lower than the expression of the T102 allele [17]. Higher frequencies of the 102C alleles of the $5-\mathrm{HT}_{2 \mathrm{~A}}$ receptor polymorphism (T102C) were found among schizophrenics [15]. Moreover, depressed patients carrying $102 \mathrm{C}$ allele had significant increased risk for suicidal behavior [2, 5]. However, there have also been several negative studies [15].

Association between gene for serotonin 2A receptor $(H T R 2 A)$ and alcoholism has been reported in prior studies $[6,8,13,16]$ with contradictory results. Nakamura et al. [16] first reported a positive association of alcohol dependence with the HTR $2 A$ gene. This work referred to the A1438G functional polymorphism in the promoter region of the gene, which is in linkage disequilibrium with $\mathrm{T} 102 \mathrm{C}$ polymorphism. The T102C (rs6313) single nucleotide polymorphism has been associated with alcoholism in male alcohol abusers as reported by Hwu and Chen [9] as well as by Lee et al. [13]. However, this association has not been observed by Fehr et al. [6]. The explanation for incongruent results can be small sample size, ethnic stratification problems or heterogeneity of diagnoses. Interestingly, in a postmortem study Underwood et al. [21] found lower 5- $\mathrm{HT}_{2 \mathrm{~A}}$ receptor binding in the prefrontal cortex (PFC) of alcoholics with a family history of alcoholism.

The above mentioned data suggest a role of the 5- $\mathrm{HT}_{2 \mathrm{~A}}$ receptor gene as a candidate gene for alcohol dependence. Therefore, we aimed to investigate whether HTR2A T102C single nucleotide polymorphism is associated with alcohol dependence, with a specific hypothesis that increased prevalence of allele $102 \mathrm{C}$ is associated with early onset of alcohol problems. The potential advantage of the current study is well-defined and homogenous group of alcoholic patients, which is representative for a population of alcohol-dependent patients in Poland.

\section{Materials and Methods}

The study included a sample of 150 patients (108 males and 42 females) admitted to addiction treatment
Tab. 1. Demographic and clinical characteristics of the alcoholdependent $(A D)$ patients

\begin{tabular}{lc}
\hline Characteristics & Participants $(n=150)$ \\
\hline Age (years); mean \pm SD & $43.27 \pm 9.7$ \\
Gender (males); $n(\%)$ & $108(72.0)$ \\
Married; $n$ (\%) & $76(50.7)$ \\
Employed; $n$ (\%) & $67(44.7)$ \\
Education (years); mean \pm SD & $11.66 \pm 3.14$ \\
Family history of alcohol dependence; $n(\%)$ & $97(64.7)$ \\
Age of onset of drinking (years); mean \pm SD & $26.17 \pm 9.69$ \\
& $23(19-31)$ \\
Michigan Alcoholism Screening Test; mean \pm SD & $33.43 \pm 9.55$ \\
\hline
\end{tabular}

SD - standard deviation

programs in Warszawa, Poland and diagnosed with alcohol dependence according to the DSM-IV criteria [1]. All subjects were Caucasian adults of a Polish nationality, unrelated to each other. The Michigan Alcoholism Screening Test (MAST) was an instrument quantifying the severity of alcohol dependence. The MAST is a self-administered 25-item questionnaire, originally designed to identify probable cases of alcoholism [18]. Patients were evaluated also with the Mini International Neuropsychiatric Interview (M.I.N.I.) a short, structured interview for both DSM-IV and ICD-10 psychiatric diagnoses [19]. Demographic data of all the patients are presented in Table 1. Ethnically matched healthy subjects from the general population (healthy blood donors) served as a control group to compare the frequencies of the genotypes. The controls were mostly unrelated young male (75\%) volunteers and none of them manifested alcohol problems. Patients were included in the study after a written informed consent. The local bioethics committee of the Medical University of Warsaw and the Institutional Review Board at the University of Michigan approved the study protocol.

Peripheral blood samples were drawn from all subjects and the DNA of white blood cells was extracted using the standard procedure. The genotype of the HTR2A T102C (rs6313) single nucleotide polymorphism (SNP) was analyzed in 150 patients and in controls. Real-time PCR (polymerase chain reaction) was performed by the LightCycler ${ }^{\circledR} 480$ instrument available from Roche Applied Science. Simple Probes, 
Tab. 2. Comparison of genotype and allele frequencies of HTR2A T102C polymorphism between alcohol-dependent (AD) patients and controls

\begin{tabular}{lcccccc}
\hline & & \multicolumn{3}{c}{ Genotype frequencies } & \multicolumn{2}{c}{ Allele frequencies } \\
\cline { 3 - 6 } & & CC & CT & TT & C & T \\
\hline AD patients & 150 & $56(37 \%)$ & $76(51 \%)$ & $18(12 \%)$ & $188(0.63)$ & $112(0.37)$ \\
Controls & 80 & $23(29 \%)$ & $37(46 \%)$ & $20(25 \%)$ & $83(0.52)$ & $77(0.48)$ \\
\hline Statistics & & & $\chi^{2}=6.66 ; \mathrm{df}=2 ; p=0.036$ & $\chi^{2}=5.02 ; \mathrm{df}=1 ; p=0.025$ \\
\hline
\end{tabular}

LightCycler ${ }^{\circledR} 480$ Probes Master Mix and universal cycling conditions were used. LightSNiP (SimpleProbe) assays for SNPs were designed by TIB-MolBiol (Berlin, Germany).

\section{Statistical analysis}

The data were analyzed with Statistica 9.0 software. All genotyping results were tested for HardyWeinberg equilibrium (HWE). For statistical analysis of the differences in the allele and genotype frequencies of the $5-\mathrm{HT}_{2 \mathrm{~A}}$ receptor gene between the alcoholic group and the control group the $\chi^{2}$ statistics was used. From this analysis, the odds ratios (ORs) with 95\% confidence intervals (CIs) were reported. To investigate the effect of the $102 \mathrm{C}$ allele on the age at onset of problems from drinking Mann-Whitney U test was applied. The significance level for all statistical tests was 0.05 .

\section{Results}

The distributions of HTR2A T102C polymorphism in all alcohol-dependent (AD) patients $\left(\chi^{2}=1.03, \mathrm{df}=1\right.$, $\mathrm{p}=0.31)$ and controls $\left(\chi^{2}=0.43, \mathrm{df}=1, \mathrm{p}=0.51\right)$ were in agreement with the Hardy-Weinberg equilibrium (HWE). In the comparison of allele and genotype frequencies, the examined polymorphism showed a significant difference between AD patients and the control group (Tab. 2). Specifically, the frequency of the $102 \mathrm{C}$ allele in the group of $\mathrm{AD}$ patients was higher than in the control group $\left(\chi^{2}=5.02 ; \mathrm{df}=1\right.$, $\mathrm{p}=0.025)$. Moreover, the group of AD subjects had lower frequencies of TT genotype and higher frequencies of the CC genotype in contrast with the normal controls $\left(\chi^{2}=6.10 ; \mathrm{df}=1 ; \mathrm{p}=0.014\right)$. The estimated odds ratio for alcohol dependence with the $\mathrm{C}$ allele was 1.56 (95\% CI: 1.06-2.30) with the effect size $\varphi=$ 0.1 for the $\chi^{2}$ test. The statistical analysis revealed a significant association between the HTR2A polymorphism and the age at onset of problems from drinking. Patients homozygous for allele 102C $(\mathrm{n}=$ 55 ) had significantly lower age at onset of problems from drinking (median: 21 years; IQR: 18-28) than subjects having at least one $\mathrm{T}$ allele in T102C polymorphism $(n=94)$ (median, 25 years; IQR: 20-35; $\mathrm{p}=0.018)$.

\section{Discussion and Conclusion}

We performed an association study with the T102C polymorphism (rs6313) in the $5-\mathrm{HT}_{2 \mathrm{~A}}$ receptor gene in a sample of $150 \mathrm{AD}$ patients and 80 healthy controls. The genetic analyses showed that AD patients had significantly lower frequencies of the T102 allele and T102T genotype in the HTR $2 A$ rs6313 polymorphism than the control group. The results are in accordance with a previous report by Hwu et al. [9] where the less frequent TT genotype was associated with alcohol abuse in male patients. Moreover, the study of Lee et al. [13] has found that the genotype and allele distribution of $\mathrm{T} 102 \mathrm{C}$ polymorphism in the 119 male alcoholics was significantly different from 70 controls (but the genotypic distribution was not in HW equilibrium in controls). On the other hand, our findings differ from the prior case-control association study by Fehr et al. [6] that found no association between the T102C polymorphism and alcoholism in the German population where allele and genotype frequencies were similar between the alcohol-dependent subjects and the healthy controls [6]. In favor of our results is the meta-analysis by Li et al. [15] of HTR2A T102C 
polymorphism in schizophrenia. Interestingly, in the 48 studies which included 7363 cases and 7311 controls, 30 studies showed lower T102 allele frequency in cases than in controls. The average frequency was $52.9 \%(33.8-67.7 \%)$ across all populations, with $46.9 \%$ in the European populations and $57.6 \%$ in the Asian samples [15]. The main cause of discrepancies between our and some previous findings may be due to the fact that genotypic and allelic frequencies differ between diverse ethnic groups. Moreover, as both the current and some previous studies $[6,9,13]$ had small sample sizes, limited power can be a possible explanation for divergent results. Future meta-analyses concerning the influence of the HTR $2 A$ T102C polymorphism on alcohol dependence in numerous groups are a potential way to reduce the problems with small samples and would allow for a final conclusion. The potential strength of the current study is that the sample is characterized by a relative genetic homogeneity, which is well established for a Polish population. Thus, our sample is representative for the population of alcohol-dependent individuals of a Polish descent.

Future studies of this polymorphism for association with alcohol dependence remain an interesting focus for investigations, especially in light of the findings suggested that the HTR $2 A$ polymorphisms might have functional effects on expression of the $5-\mathrm{HT}_{2 \mathrm{~A}}$ receptor in the brain [21]. Increased prevalence of $102 \mathrm{C}$ allele among alcoholics and intrinsically low expression of this allele may contribute to decreased total levels of $5-\mathrm{HT}_{2 \mathrm{~A}}$ receptors $\mathrm{mRNA}$ and protein. Due to that mechanism, patients carrying HTR2A 102C allele may have higher susceptibility to develop alcohol addiction than non-carriers. Accordingly, in this study patients homozygous for $\mathrm{C}$ allele had significantly earlier age at onset of problems from drinking than subjects having at least one $\mathrm{T}$ allele in $\mathrm{T} 102 \mathrm{C}$ polymorphism ( $p=0.018)$. This is particularly important in order to identify individuals with increased risk of severe course of alcohol dependence and more frequent consequences. Alcoholics with early onset of a disease have worse prognosis and poorer treatment outcomes. Moreover, in our previous work it has been speculated that the HTR $2 A$ T102C polymorphisms might influence the course of alcoholism due to influence on accompanying symptoms like impulsivity and suicide behaviors [10, 22]. Using genetic typing of alcoholic subjects could allow in future supplementing current typologies of alcohol dependence with genetic characteristics if future studies would confirm that early onset of alcoholism is associated with the HTR2A T102C polymorphism. However, more research is needed on larger samples to allow for reliable conclusions.

The present study has several limitations, which should be taken into account. First, it is possible that due to the small sample size our study had insufficient statistical power to detect associations with a small effect size. Moreover, the study used unmatched cases of controls and alcoholics, thereby not specifically controlling for variables such as age and sex. Lack of diagnosing blood donors for psychiatric disorders and corroboration of their self-report of substance use we treat as another restriction of our study. Finally, in the population of alcohol-dependent patients included in our study the diagnostic procedures did not type subjects into subtypes of alcohol dependence in any of the current classifications (e.g., Cloninger, Lesch, Babor).

\section{Acknowledgments:}

This study was supported by the Polish Ministry of Science and Higher Education grant (MNiSW NN 405357 239), the Fogarty International Center/NIDA International Substance Abuse Research Program grant D43-TW05818, the Fogarty International Center/NIAAA International Collaborative Alcohol \& Injury Research Training Program grant D43-TW007569 and NIAAA grant R21 AA016104

\section{References:}

1. American Psychiatric Association: Diagnostic and statistical manual of mental disorders, 4th edn. - Text Revision (DSM IV-TR), Washington, D.C., 2000.

2. Arias B, Gastó C, Catalán R, Gutiérrez B, Pintor L, Fañanás L: The $5-\mathrm{HT}_{2} \mathrm{~A}$ receptor gene $102 \mathrm{~T} / \mathrm{C}$ polymorphism is associated with suicidal behavior in depressed patients. Am J Med Genet, 2001, 105, 801-804.

3. Crawford LK, Craige CP, Beck SG: Increased intrinsic excitability of lateral wing serotonin neurons of the dorsal raphe: a mechanism for selective activation in stress circuits. J Neurophysiol, 2010, 103, 2652-2663.

4. Dick DM, Foroud T: Candidate genes for alcohol dependence: a review of genetic evidence from human studies. Alcohol Clin Exp Res, 2003, 27, 868-879.

5. Du L, Bakish D, Lapierre YD, Ravindran AV, Hrdina PD: Association of polymorphism of serotonin 2A receptor gene with suicidal ideation in major depressive disorder. Am J Med Genet, 2000, 96, 56-60.

6. Fehr C, Schleicher A, Szegedi A, Anghelescu I, Klawe C, Hiemke C, Dahmen N: Serotonergic polymorphisms in patients suffering from alcoholism, anxiety disorders and narcolepsy. Prog Neuropsychopharmacol Biol Psychiatry, 2001, 25, 965-982. 
7. Haleem DJ: Behavioral deficits and exaggerated feedback control over raphe-hippocampal serotonin neurotransmission in restrained rats. Pharmacol Rep, 2011, 63, 888-897.

8. Huang CL: The role of serotonin and possible interaction of serotonin-related genes with alcohol dehydrogenase and aldehyde dehydrogenase genes in alcohol dependence - a review. Am J Transl Res, 2010, 2, 190-199.

9. Hwu HG, Chen CH: Association of 5HT2A receptor gene polymorphism and alcohol abuse with behavior problems. Am J Med Genet, 2000, 96, 797-800.

10. Jakubczyk A, Wrzosek M, Łukaszkiewicz J, SadowskaMazuryk J, Matsumoto H, Śliwerska E, Glass J et al.: The CC genotype in HTR2A T102C polymorphism is associated with behavioral impulsivity in alcoholdependent patients. J Psychiatr Res, 2012, 46, 44-49.

11. Lanzenberger R, Wadsak W, Spindelegger C, Mitterhauser M, Akimova E, Mien LK, Fink M et al.: Cortisol plasma levels in social anxiety disorder patients correlate with serotonin-1A receptor binding in limbic brain regions. Int J Neuropsychopharmacol, 2010, 13, 1129-1143.

12. Lanzenberger RR, Mitterhauser M, Spindelegger $C$, Wadsak W, Klein N, Mien LK, Holik A et al.: Reduced serotonin-1A receptor binding in social anxiety disorder. Biol Psychiatry, 2007, 61, 1081-1089.

13. Lee YS, Choi SW, Han DH, Kim DJ, Joe KH: Clinical manifestation of alcohol withdrawal symptoms related to genetic polymorphisms of two serotonin receptors and serotonin transporter. Eur Addict Res, 2009, 15, 39-46.

14. Lesch KP: Alcohol dependence and gene $\mathrm{x}$ environment interaction in emotion regulation: Is serotonin the link? Eur J Pharmacol, 2005, 526, 113-124.

15. Li D, Duan Y, He L: Association study of serotonin 2A receptor (5-HT2A) gene with schizophrenia and suicidal behavior using systematic meta-analysis, Biochem Biophys Res Commun, 2006, 340, 1006-1015.
16. Nakamura T, Matsushita S, Nishiguchi N, Kimura M, Yoshino A, Higuchi S: Association of a polymorphism of the 5-HT2A receptor gene promoter region with alcohol dependence. Mol Psychiatry, 1999, 4, 85-88.

17. Polesskaya OO, Sokolov BP: Differential expression of the " $C$ " and " $T$ " alleles of the 5-HT2A receptor gene in the temporal cortex of normal individuals and schizophrenics. J Neurosci Res, 2002, 67, 812-822.

18. Selzer ML: The Michigan alcoholism screening test: the quest for a new diagnostic instrument. Am J Psychiatry, 1971, 127, 1653-1658.

19. Sheehan DV, Lecrubier Y, Sheehan KH, Amorim P, Janavs J, Weiller E, Hergueta T et al.: The Mini-International Neuropsychiatric Interview (M.I.N.I.): the development and validation of a structured diagnostic psychiatric interview for DSM-IV and ICD-10. J Clin Psychiatry, 1998, 59, Suppl 20, 22-33.

20. Stokes PE: The potential role of excessive cortisol induced by HPA hyperfunction in the pathogenesis of depression. Eur Neuropsychopharmacol, 1995, 5, Suppl 1, 77-82.

21. Underwood MD, Mann JJ, Huang YY, Arango V: Family history of alcoholism is associated with lower 5-HT2A receptor binding in the prefrontal cortex. Alcohol Clin Exp Res, 2008, 32, 593-599.

22. Wrzosek M, Łukaszkiewicz J, Wrzosek M, Serafin P, Jakubczyk A, Klimkiewicz A, Matsumoto $\mathrm{H}$ et al.: Association of polymorphisms in HTR2A, HTR1A and TPH2 genes with suicide attempts in alcohol dependence: A preliminary report. Psychiatry Res, 2011, 190, 149-151.

Received: July 22, 2011; in the revised form: November 8, 2011; accepted: December 5, 2011. 